Article

\title{
The Effect of Primary Duodenogastric Bile Reflux on the Presence and Density of Helicobacter pylori and on Gastritis in Childhood
}

\author{
Mehmet Agin ${ }^{1, *(1)}$ and Yusuf Kayar ${ }^{2}$ \\ 1 Department of Pediatric, Division of Pediatric Gastroenterology, Hepatology and Nutrition, Van Education \\ and Research Hospital, 65300 Van, Turkey \\ 2 Department of Internal Medicine, Division of Gastroenterology and Hepatology, Van Education and \\ Research Hospital, 65300 Van, Turkey; ykayar@yahoo.com \\ * Correspondence: drmehmet47@yahoo.com; Tel.: +90-50-6801-1083; Fax: +90-0-432-2175-600
}

Received: 25 September 2019; Accepted: 2 December 2019; Published: 5 December 2019

\begin{abstract}
Background and Objectives: Although there are many studies that investigate the relationship between duodenogastric reflux (DGR) and Helicobacter pylori in adult patients, the reported data are contradictory. In addition, there are very few studies in the literature investigating the relationship between DGR and H. pylori in the pediatric age group. In the present study, we investigated the effect of primary DGR on H. pylori and gastritis. Materials and Methods: A total of 361 patients who were referred to the clinic of our hospital with dyspeptic complaints who had an upper gastrointestinal system endoscopy and a gastric biopsy were included in the study. Results: DGR was detected in 45 cases, and 316 cases that did not have DGR were considered as the control group. Comparisons were made between the DGR cases and the control group in terms of risk factors (age, gender), the presence and density of $\mathrm{H}$. pylori, and the presence and severity of gastritis. The average age of the patients who were included in the study was $11.6 \pm 4.6$ years. A total of $128(36 \%)$ of the cases were male and $233(64 \%)$ were female. DGR was present in $45(13 \%)$ of the cases. The average age of the patients with DGR was $13.9 \pm 3.1$ years, the average age of the control group was $11.3 \pm 4.7$, and there were statistically significant differences $(p<0.001)$. No significant differences were detected in terms of gender between DGR and the control group $(p>0.05)$. H. pylori $(+)$ was detected in $29(64 \%)$ of patients with DGR, and in $202(64 \%)$ of the control group. No significant differences were detected between $H$. pylori prevalence $(p=0.947)$. Gastritis was detected in $37(82 \%)$ of the patients with DGR, and in $245(77 \%)$ of the control group ( $p=0.476)$. No significant differences were detected between the presence and density of $H$. pylori, gastritis presence, severity and DGR $(p>0.05)$. Conclusions: The ages of patients with DGR were significantly higher than in the control group, and advanced age was shown to be a risk factor for primary DGR. It was found that the presence of DGR has no effect on the presence and severity of $H$. pylori. Given this situation, we consider it is important to eradicate $H$. pylori infection, especially in the case where H. pylori is present together with DGR.
\end{abstract}

Keywords: duodenogastric bile reflux; gastritis; Helicobacter pylori; age

\section{Introduction}

Duodenogastric reflux (DGR) is the passing of the duodenal contents from the duodenum into the stomach [1]. Although DGR is quite common after cholecystectomy, pyloroplasty and gastric surgery, it can also develop primarily because of pyloric insufficiency without any secondary causes $[1,2]$. It was shown in previous studies that there might be a small amount of DGR physiologically in the stomach after feeding and in fasting [2,3]. The most important factor in the re-escape of the duodenum contents into the stomach is the deterioration in pylori function [4]. Although gastric acid and pepsin 
are the primary gastric agents providing the basis for mucosal damage and esophageal symptoms in the esophagus, damage occurs in the stomach and esophagus mucosa as a result of bile reflux and in the pancreas and with small intestine secretions from the duodenum into the stomach. Watt et al. showed that bile reflux into the stomach was associated with gastric ulcers and gastritis in patients who had dysfunction in the pyloric sphincter [5]. However, it is known that clinical complaints are disproportionate to mucosal damage. It was shown experimentally that DGR induced gastritis is not always symptomatic. Although complaints like epigastric pain, pain in the retrosternal region, and vomiting bile may develop after DGR, the presence and severity of symptoms were not found to be proportional to the amount of bile in the reflux [6,7]. Although DGR does not have a gold standard diagnosis, the presence of ulcers, erosion, fragility, mucosal erythema, and an abundant bile pool in the stomach seen in an endoscopy (especially in patients who have abdominal pain), epigastric pain, nausea and vomiting are characteristics of DGR [8-10].

Helicobacter pylori is a spiral-shaped Gram-negative bacterium that colonizes the gastric mucosa. H. pylori is the most common cause of resistant bacterial infection in the world and is located in the gastric mucosa and causes gastritis, peptic ulcers, B cell lymphoma and gastric cancer [11,12]. Although DGR and $H$. pylori cause different histopathological changes, $H$. pylori disrupts the mucosal barrier, initiates inflammation and sets the stage for an ulcer. Although there have been many studies to investigate the relationship between $H$. pylori and DGR in the adult age group, the data are contradictory $[13,14]$. In some studies, it was argued that the mucus barrier had deteriorated in the gastric mucosa because of increased bile acid, and as a result of this, H. pylori colonization decreases [13]. There are also other studies reporting that bile reflux increases gastric $\mathrm{pH}$, disrupts the microenvironment needed for the survival of $H$. pylori, and eradicates it by disrupting colonization $[13,14]$. However, it was reported in some studies that there is a positive relationship between $H$. pylori and DGR, and DGR increased the presence of $H$. pylori $[15,16]$. There are no studies in Turkey that investigate the relationship between primary DGR and H. pylori in the childhood age group. In this study, we aimed to determine the relationship between primary DGR with the presence and density of $H$. pylori and gastritis, and the risk factors that affect DGR development.

\section{Material and Methods}

\subsection{Patient Selection}

A total of 361 cases who presented to the Pediatric Gastroenterology Clinic of the University of Health Sciences Van Education and Research Hospital between October 2017 and December 2018 with dyspeptic complaints who had undergone an upper gastrointestinal(GI) system endoscopy and gastric biopsy were included in the study. DGR was detected in 45 cases, and the 316 non-DGR cases were used as the control group. The age, gender, history, family history, history of metabolic disease, presence of concomitant systemic disease, upper GI system endoscopy results, histopathology results of the biopsies taken from the gastric mucosa, whether they were receiving $H$. pylori eradication therapy and abdominal ultrasonography (USG) data of the patients were recorded. In our study, the age, gender, family history, past surgical and interventional procedures, and the presence of pancreatic, hepatobiliary system and GI system pathologies seen in the abdominal USG were evaluated to determine the DGR risk factors. Those who underwent prior $H$. pylori eradication treatments, prior surgery for gastritis, prior gastric surgery, prior bile and/or bile duct surgery, prior endoscopic retrograde cholangiopancreatography (ERCP) procedures, those who received steroid and non-steroid anti-inflammatory treatment, those with diabetes, and those who had systemic comorbid diseases such as hypertension were excluded from the study.

\subsection{Endoscopic Evaluation}

The endoscopies of the patients were carried out by using a EG530WR Endoscopy (Fujinon, Tokyo, Japan) Device in the Endoscopy Unit of the Van Education and Research Hospital. Oral and 
written consent was received from the families before the endoscopy. All patients fasted for $6 \mathrm{~h}$ before the endoscopy, and after local pharyngeal xylocaine anesthesia, endoscopic procedures were carried out after the patients were sedated with midazolam $(0.1 \mathrm{mg} / \mathrm{kg})$ and ketamine $(1 \mathrm{mg} / \mathrm{kg})$. During the endoscopy, the esophagus, cardia, fundus, corpus and antrum regions of the stomach, and the duodenum were examined in detail. During the endoscopic examination, gastritis findings such as hyperemia, fragility, edema, gastric and duodenal ulcers and erosions, masses, hemorrhage, hiatal hernia, strictures and stenosis in the inferior esophageal sphincter, bile pool, and duodenogastric bile reflux were noted. The endoscopic data of the patients were recorded.

\subsection{Histopathologic Evaluation}

The endoscopic corpus and antrum biopsies were sent to the pathology laboratory in $10 \%$ formaldehyde. After the routine tissue follow-up procedures, the tissue samples that were embedded in paraffin were cut into 5-micron-thick slices, stained with routine hematoxylin-eosin (H-E), and evaluated under a light microscope. Following a rapid urease test to confirm the diagnosis of H. pylori, the biopsies were stained with modified Giemsa to histopathologically assess $H$. pylori presence and density. We measured the presence and density of $H$. pylori in patient biopsies, and the presence and severity of gastritis in accordance with the modified Sydney classification. Biopsies were assessed for the intensity of mononuclear inflammatory cellular infiltrates, inflammatory activity (neutrophilic infiltrations), glandular atrophy, dysplasia, metaplasia, and reparative atypia. Moreover, cases were graded according to the Houston and updated Sydney system, where the grading was in accordance with the intensity of mononuclear inflammatory cellular infiltrates within the lamina propria: absent inflammation (Grade 0), mild inflammation (Grade 1), moderate inflammation (Grade 2), and severe inflammation (Grade 3). The presence of H. pylori was interpreted as $1(+), 2(+)$ or $3(+)$, and the severity of gastritis was interpreted as mild, moderate or severe $[17,18]$.

\subsection{Ethical Board Approval}

Verbal and written informed consent was obtained from all the subjects included in the study and from their parents. After the study was completed, the study results for each subject was reported to their parents. Ethics committee approval for the study was given by the Van Education and Research Hospital Clinical Research Ethics Committee (Van/Turkey, Approval number 01/13, approved on 5 May 2019).

\subsection{Statistical Analysis}

The results of our study were analyzed with the Statistical Package for Social Sciences v.19.0 (SPSS Armonk, NY, USA: IBM Corp.) program. The data that had continuous values were given as average (mean \pm standard deviation (SD)), and the categorical data were given as frequency and percentage $(n, \%)$. The data were tested for compliance in terms of normal distribution with the Kolmogorov-Simirnov test, histograms and \pm SD. The Mann-Whitney Utest, Shaphiro-Wilk test and Student $t$-test were used for the analysis of non-parametric data of the groups, and the Chi-Square Test was used to analyze the categorical data. $p<0.05$ was considered to be statistically significant.

\section{Results}

A total of 361 patients were included in the present study. The average age of the cases was $11.6 \pm 4.6$ years. A total of $128(36 \%)$ of the cases were male, and $233(64 \%)$ were female. DGR was detected in 45 cases $(13 \%)$, and not detected in 316 patients. Five cases with DGR (11\%), and 31 non-DGR cases $(10 \%)$ had kinship between parents. There was no statistically significant difference between the two groups. None of the cases had a family history of DGR, metabolic disease, prior gastric surgery, prior bile and/or bile duct surgery or prior ERCP. In all cases, H. pylori prevalence was $64 \%(231 / 361)$. In the evaluation of $H$. pylori density, the results were $1(+)$ in 112 patients, $2(+)$ in 80 patients, and $3(+)$ in 32 patients. Gastritis was detected in $78 \%(282 / 361)$ of patients. In the 
evaluation made for gastritis severity, the results were mild in 135 patients, moderate in 112 patients, and severe in 35 patients (Table 1).

Table 1. The demographic, endoscopic and histopathologic data of the patients.

\begin{tabular}{cccc}
\hline \multirow{2}{*}{ Gender } & & Number & \% \\
\hline \multirow{2}{*}{ Duodenogastric bile reflux } & Male & 128 & 36 \\
& Female & 233 & 64 \\
\hline \multirow{2}{*}{ Helicobacter pylori } & Yes & 45 & 13 \\
& No & 316 & 87 \\
\hline \multirow{2}{*}{ Helicobacter pylori density } & Yes & 231 & 64 \\
& No & 130 & 36 \\
\hline \multirow{2}{*}{ Gastritis } & 1 & 112 & 31 \\
& 2 & 80 & 22 \\
& 3 & 39 & 11 \\
\hline \multirow{2}{*}{ Gastritis severity } & Yes & 282 & 78 \\
& No & 79 & 22 \\
\hline & Mild & 135 & 37 \\
& Moderate & 112 & 31 \\
& Severe & 35 & 10 \\
\hline
\end{tabular}

Patients who had DGR were compared with the control group (without DGR) to evaluate the effect of DGR on the prevalence and density of H. pylori and the presence and severity of gastritis. The average age of the patients who had DGR was $13.9 \pm 3.1$ years, and the average age of the control group was $11.3 \pm 4.7$ years; it was determined that the difference was statistically significant $(p<0.001)$. When the relationship between gender and DGR was examined, it was determined that $15(33 \%)$ of the patients with DGR were male, the number of the males in the control group was $113(36 \%)$, and the difference was not at a significant level $(p>0.05)$. When the relationship between DGR and H. pylori was examined, it was determined that $29(64 \%)$ of the patients who had DGR were H. pylori (+), and $202(64 \%)$ of the control group were H. pylori $(+)$. In the group with DGR, H. pylori density was $1(+)$ in $13(29 \%)$ patients, $2(+)$ in eight $(18 \%)$ patients, and $3(+)$ in eight $(18 \%)$ patients; in the patients in the control group, the H. pylori density was $1(+)$ in $99(31 \%)$ patients, $2(+)$ in $72(23 \%)$ patients, and $3(+)$ in $31(10 \%)$ patients. No significant differences were detected between the two groups in terms of $H$. pylori prevalence or $H$. pylori density ( $p=0.947, p=0.244$, respectively). When the relationship between DGR and gastritis was analyzed, it was determined that gastritis was detected in $37(82 \%)$ of cases with DGR, and in 245 (77\%) of the control group. In the group that had DGR, gastritis severity was mild in $19(51 \%)$ patients, moderate in $13(35 \%)$ patients, and severe in five $(14 \%)$ patients. In the control group, gastritis severity was mild in $116(47 \%)$ patients, moderate in $99(40 \%)$ patients, and severe in 30 $(12 \%)$ patients. No significant differences were detected in terms of presence and severity of gastritis between the two groups ( $p=0.476, p=0.829$, respectively) (Table 2). Logistic regression analysis was performed on all patients. We found that duodenogastric bile reflux had no effect on the severity of gastritis in the gastric mucosa, but that the severity of gastritis increased by 15.4 times in the presence of $H$. pylori. The frequency of H. pylori and gastritis in the DGR and control (non-DGR cases) groups is shown in Figure 1. 
Table 2. The relationship between DGR presence and demographic data, presence and density of $H$. pylori, and presence and severity of gastritis.

\begin{tabular}{|c|c|c|c|c|}
\hline & & \multicolumn{3}{|c|}{ Duodenogastric Bile Reflux } \\
\hline & & Yes & No & $p$ \\
\hline \multicolumn{2}{|c|}{ Age } & $13.9 \pm 3.1$ & $11.3 \pm 4.7$ & $<0.001$ \\
\hline \multirow{2}{*}{ Gender } & Male & $15(33 \%)$ & $113(36 \%)$ & \multirow{2}{*}{0.750} \\
\hline & Female & $30(67 \%)$ & $203(64 \%)$ & \\
\hline \multirow{2}{*}{$\begin{array}{c}\text { Presence of } \\
\text { Helicobacter pylori }\end{array}$} & Yes & $29(64 \%)$ & $202(64 \%)$ & \multirow{2}{*}{0.946} \\
\hline & No & $16(36 \%)$ & $114(36 \%)$ & \\
\hline \multirow{3}{*}{$\begin{array}{c}\text { Density of } \\
\text { Helicobacter pylori }\end{array}$} & 1 & $13(29 \%)$ & $99(31 \%)$ & \multirow{3}{*}{0.244} \\
\hline & 2 & $8(18 \%)$ & $72(23 \%)$ & \\
\hline & 3 & $8(18 \%)$ & $31(10 \%)$ & \\
\hline \multirow{2}{*}{ Gastritis } & Yes & $37(82 \%)$ & $245(78 \%)$ & \multirow{2}{*}{0.476} \\
\hline & No & $8(18 \%)$ & $71(23 \%)$ & \\
\hline \multirow{3}{*}{ Gastritis severity } & Mild & $19(51 \%)$ & $116(47 \%)$ & \multirow{3}{*}{0.829} \\
\hline & Moderate & $13(35 \%)$ & $99(40 \%)$ & \\
\hline & Severe & $5(14 \%)$ & $30(12 \%)$ & \\
\hline
\end{tabular}

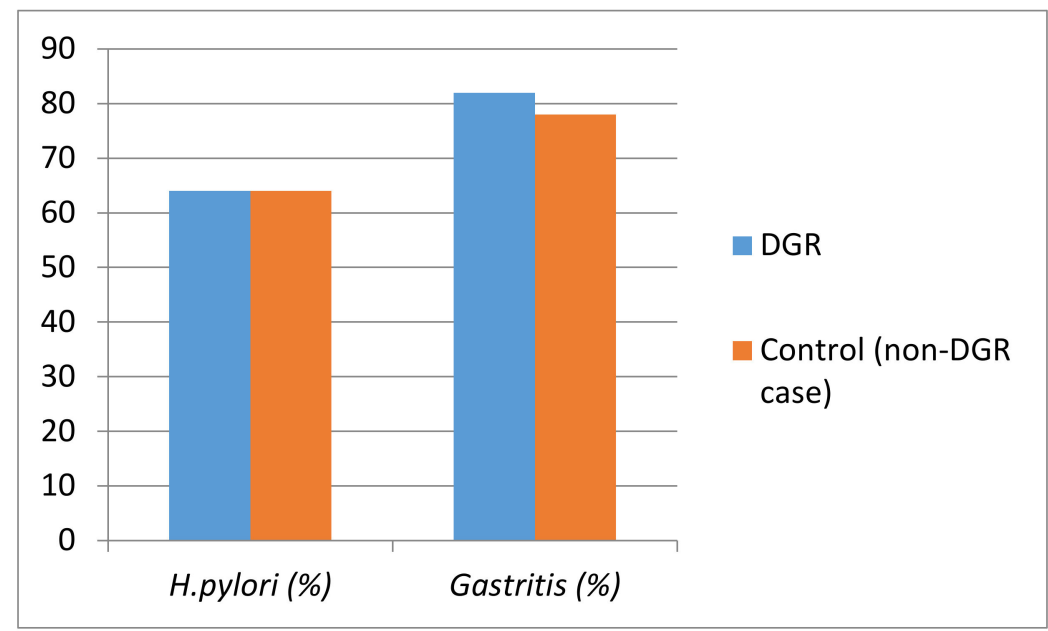

Figure 1. Comparison of the frequency of H. pylori and gastritis in the duodenogastric reflux (DGR) and control (non-DGR case) groups.

\section{Discussion}

Although there are wide-scale studies that investigate the relationship between DGR and H. pylori gastritis due to secondary causes, there is ascarcity of studies in which the relation between primary DGR and H. pylori gastritis is analyzed [19]. In our study, we aimed to investigate the relationship between primary DGR development, demographic data and H. pylori gastritis. In addition, age, gender, kinship, family history, metabolic disease, surgical and interventional procedures in their histories and the presence of pancreatic, hepatobiliary system and GI system pathologies in abdominal USG were evaluated to determine DGR risk factors. There were no other risk factors associated with DGR other than age. It was demonstrated that DGR was higher at a significant level in the elderly; however, no significant correlation was detected between DGR development and gender, density and presence of $H$. pylori, and presence and severity of gastritis. 
In this study, we demonstrated that DGR was significantly higher in older children. The mean age of children with DGR was $13.9 \pm 3.1$ years, while the mean age of children without DGR was $11.3 \pm 4.7$ years. We did not find any previous studies on this subject in the pediatric age group. Previous studies in the adult age group have reported that the course of primary DGR is bimodal in terms of age and is more common in the younger age group (age range: 21-30 years) but is also common in the 50-80 year age range $[19,20]$. In our study, we found that there was no significant relationship between DGR and gender and that it was seen equally in both genders. We did not find any studies investigating the relationship between gender and DGR in the child population. Although there is one study examining the relationship between DGR and gender in the adult population, there are studies showing that it is 1.5:1 higher in females:males [21]. Studies have reported that $H$. pylori gastritis is seen equally in both genders because of the same probability of being affected by H. pylori infection, but DGR may be more common in women due to functional pathophysiology [19]. Mercan et al. reported that there was no significant gender difference between groups with and without DGR [22]. The results obtained from our study support this finding.

In our study, we found that DGR did not affect either the presence or severity of gastritis. However, it is still unclear whether primary DGR causes histological changes in the gastric mucosa in children $[23,24]$. It has been reported that short-term reflux of the duodenal contents into the stomach during physiological events can rarely cause symptoms. DGR is due to excessive reflux of bile, pancreas secretions and intestinal secretions into the stomach. An increased bile reflux rate may lead to increased gastric mucosal damage [23]. Biliary reflux secondary to surgery has characteristic histopathological changes such as foveolar hyperplasia, edema, hyperplasia in the lamina propria, and mild inflammation [23]. Wang et al. demonstrated that mild chronic inflammation occurred after prolonged reflux in their study in rats [24]. Due to DGR exposure, gastric polyps are present in studies reporting severe mucosal inflammation and intestinal metaplasia [25]. Taskin et al. found no significant difference in chronic inflammation between patients with and without DGR [26]. This study supports our data.

In our study, no statistically significant difference was found between the groups with and without DGR in terms of $H$. pylori prevalence or $H$. pylori density. Although both DGR and H. pylori infection are thought to damage the gastric mucosa, the relationship between DGR and H. pylori remains controversial. Although DGR has been reported to reduce the prevalence of H. pylori in some studies, other studies have reported a synergistic effect between DGR and H. pylori, while others still reported no significant relationship between them. Itoh et al. reported that bile acids had anti-bacteriological activity against $H$. pylori in vitro [27]. In previous studies, it was reported that bile inhibits $H$. pylori colonization by disrupting the microenvironment necessary for $H$. pylori colonization via disruption of the mucosal barrier and by forming an alkaline medium in the stomach, and it is concluded that bile eradicates $H$. pylori by this mechanism [13]. Sobala et al. [28] reported a negative correlation between the presence of DGR and the prevalence of H. pylori. In contrast to these data, Adam et al. in their study including 1120 pediatric patients found the prevalence of DGR to be $8.2 \%$ and the prevalence of $\mathrm{H}$. pylori to be $25 \%$; they also reported that $H$. pylori and DGR exhibited a synergistic effect [29]. Similarly, Ladas et al. reported that $H$. pylori could increase the toxic effects of DGR in intact gastric mucosa and that DGR was more frequent in $H$. pylori (+) cases and induced chronic gastritis with its synergistic effect [16]. However, no correlation was found between H. pylori intensity and DGR severity in either study. In contrast to these studies, Tewari et al. found no difference in the prevalence of $H$. pylori between patients with non-ulcer dyspepsia and biliary reflux gastritis [30]. Matsuhisa et al. also found the prevalence of $H$. pylori to be $27.2 \%$ and found no relationship between the presence or absence of DGR and the prevalence of $H$. pylori [31]. Taskin et al. also found no difference in H. pylori prevalence between patients with and without DGR [26]. Recent studies support our findings. The reason for the different results obtained in these studies can be due to the small number of cases evaluated in these studies, the presence of gastric surgery history, and differences in the amount and duration of duodenal reflux contents. Moreover, since bilirubin, which is regurgitated into the stomach, 
must act at the appropriate concentration and time to inhibit $H$. pylori infection, the present differences may be influential in the differences in outcomes [32].

Although there are strong aspects of our study, there are also some limitations. The strong aspects include: (a) investigating the age, gender, family history, parental kinship, metabolic disease, comorbid systemic diseases, anatomical anomalies and pathologies in terms of risk factors that may cause DGR through imaging; (b) evaluation of DGR in connection with the control group to examine its effects on H. pylori infection and the gastric mucosa; and (c) histopathological evaluation and comparison of H. pylori density and gastritis severity. However, only the fasting bile reflux was evaluated in our study. The fact that postprandial bile reflux and fasting bile reflux were not evaluated, and that the duration and amount of bile reflux to the stomach were not measured, are among the important limitations of our study.

\section{Conclusions}

The ages of the patients with DGR were higher at a significant level compared to those of the control group, and it was demonstrated that advanced age is a risk factor for primary DGR. It was also demonstrated that there was no significant difference between the cases with DGR and the control group in terms of the presence and density of $H$. pylori and gastritis presence and severity. However, it is already known that histopathological changes occur due to gastic mucosa exposure to bile acid and bile reflux, as well as the damage to the gastric mucosa due to $H$. pylori infection. Considering this, we believe that it is important to eradicate H. pylori infection, especially in the presence of DGR.

Author Contributions: M.A. and Y.K. developed the study protocol, screened and enrolled the patients, assessed the outcomes, preliminarily analyzed the data, and wrote the manuscript. M.A. and Y.K. developed the study protocol and analytical framework for the study and contributed to the writing of the manuscript. M.A. screened the patients. M.A. and Y.K. supervised the design and execution of the study, performed the final data analyses, and contributed to the writing of the manuscript. All the authors have read and approved the final manuscript.

Funding: This research received no external funding.

Acknowledgments: The authors declare that this study has received no financial support.

Conflicts of Interest: The authors declare no conflicts of interest.

\section{References}

1. Fein, M.; Fuchs, K.H.; Bohrer, T.; Freys, S.M.; Thiede, A. Fiberoptic technique for 14-hour bile reflux monitoring. Dig. Dis. Sci. 1996, 41, 216-225. [CrossRef] [PubMed]

2. Keane, F.B.; Dimagno, E.P.; Malagelada, J.R. Duodenogastric reflux in humans: Its relationship to fasting antroduodenal motility and gastric, pancreatic and biliary secretion. Gastroenterology 1981, 81, 726-731. [CrossRef]

3. Miiller-Lissner, S.A.; Fimmel, C.J.; Sonnenberg, A.; Will, N.; Müller-Duysing, W.; Heinzel, F.; Müller, R.; Blum, A.L. Novel approach to quantity duodenogastric reflux in healthy volunteers and in patients with type I gastric ulcus. Gut 1983, 24, 515-517.

4. Pazzi, P.; Scalia, S.; Stabellini, G.; Trevisani, L.; Alvisi, V.; Guarneri, M. Bile reflux gastritis in patients without prior gastric surgery: Therapeutic effects of ursodeoxycholic asid. Curr. Ther. Res. 1989, 45, 476-487.

5. Watt, P.C.H.; Sloan, J.M.; Spencer, A.; Kennedy, T.L. Histology of the postoperative stomach before and after diversion of bile. Br. Med. J. (Clin. Res. Ed.) 1983, 287, 1410-1412. [CrossRef] [PubMed]

6. Hermans, D.; Sokal, E.M.; Collard, J.M.; Romagnoli, R.; Buts, J.P. Primary duodenogastric reflux in children and adolescents. Eur. J. Pediatr. 2003, 162, 598-602. [CrossRef]

7. Ortiz, P.; Santibanez, G.; Briones, E.; Marín, V.; Palma, A. Duodenogastric reflux: Validation study of its endoscopic visualization. Rev. Med. Chile. 1998, 126, 279-283.

8. Lee, E.L.; Feldman, M. Gastritis and gastropathies. In Sleisenger and Fordtran's Gastrointestinal and Liver Diseases, 8th ed.; Feldman, M., Friedman, L., Brandt, L.J., Eds.; Saunders Elsevier: Philadelphia, PA, USA, 2006; pp. 1067-1083. 
9. Loffeld, R.J.; Liberov, B.; Dekkers, P.E. The changing prevalence of upper gastrointestinal endoscopic diagnoses: A single-centre study. Neth. J. Med. 2012, 70, 222-226.

10. Shih, W.J.; Shih, G.; Milan, P.P.; Chang, C.Y.; Huang, W.S. Intrathoracic gastric activity on cholescintigraphy results in a large hiatus hernia with duodenogastric reflux. Clin. Nucl. Med. 2010, 35, 376-378. [CrossRef]

11. Pellicano, R.; Ribaldone, D.G.; Fagoonee, S.; Astegiano, M.; Saracco, G.M.; Megraud, F. A 2016 panorama of Helicobacter pylori infection: Key messages for clinicians. Panminerva Med. 2016, 58, 304-317.

12. Miciuleviciene, J.; Calkauskas, H.; Jonaitis, L.; Kiudelis, G.; Tamošiūnas, V.; Praškevičius, A.; Kupčinskas, L.; Berg, D. Helicobacter pylori genotypes in Lithuanian patients with chronic gastritis and duodenal ulcer. Medicina 2008, 44, 449-454. [CrossRef] [PubMed]

13. O'Connor, H.J.; Wyatt, J.I.; Dixon, M.F.; Axon, A.T. Campylobacter like organisms and reflux gastritis. J. Clin. Pathol. 1986, 39, 531-534. [CrossRef] [PubMed]

14. Niemela, S.; Karttunen, T.; Heikkla, J.; Mäentausta, O.; Lehtola, J. Relationship of Campylobacter pylori and duodenogastric reflux. Dig. Dis. Sci. 1989, 34, 1021-1024. [CrossRef] [PubMed]

15. Netzer, P.; Gut, A.; Brundler, R.; Gaia, C.; Halter, F.; Inauen, W. Influence of pantoprazole on oesophageal motility, and bile and acid reflux in patients with oesophagitis. Aliment. Pharmacol. Ther. 2001, 15, 1375-1384. [CrossRef]

16. Ladas, S.D.; Katsogridakis, J.; Malamou, H.; Giannopoulou, H.; Kesse-Elia, M.; Raptis, S.A. Helicobacter pylori mayinduce bile reflux: Link between $\mathrm{H}$ pylori and bile-induced injury to gastric epithelium. Gut 1996, 38, 15-18. [CrossRef]

17. Dixon, M.F.; Genta, R.M.; Yardley, J.H.; Gorrea, P. Classification and grading of gastritis. The updated Sydney system. International Workshop on the Histopathology of Gastritis, Houston 1994. Am. J. Surg. Pathol. 1996, 20, 1161-1181. [CrossRef]

18. Dixon, M.F.; Genta, R.M.; Yardley, J.H.; Gorrea, P. Histological classification of gastritis and Helicobacter pylori infection: An agreement at last. The International Workshop on the Histopathology of Gastritis? Helicobacter 1997, 2, S17-S24. [CrossRef]

19. Barakat, E.A.; Abbas, N.F.; El-Kholi, N.Y. Primary bile reflux gastritis versus Helicobacterpylori gastritis: A comparative study. Egypt. J. Intern. Med. 2018, 30, 23-27. [CrossRef]

20. Vere, C.C.; Cazacu, S.; Comănescu, V.; Mogoantă, L.; Rogoveanu, I.; Ciurea, T. Endoscopical and histological features in bile reflux gastritis. Rom. J. Morphol. Emberyol. 2005, 46, 269-274.

21. Kellosalo, J.; Alavaikko, M.; Laitinen, S. Effect of biliary tract procedures on duodenogastric reflux and the gastric mucosa. Scand. J. Gastroenterol. 1991, 26, 1272-1278. [CrossRef]

22. Mercan, E.; Duman, U.; Tihan, D.; Dilektasli, E.; Senol, K. Cholecystectomy and duodenogastric reflux: Interacting effects over the gastric mucosa. SpringerPlus 2016, 5, 1970. [CrossRef] [PubMed]

23. Zhang, Y.; Yang, X.; Gu, W.; Shu, X.; Zhang, T.; Jiang, M. Histological features of the gastric mucosa in children with primary bile reflux gastritis. World J. Surg. Oncol. 2012, 10, 27. [CrossRef] [PubMed]

24. Wang, X.W.; Mo, J.Z.; Zhi, L.M.; Chen, X.Y.; Shi, Y.; Xiao, S.D. The influence of longterm bile reflux on rat gastric mucosa. Chin. J. Dig. 2006, 26, 295-299.

25. Chang, W.K.; Lin, C.K.; Chuan, D.C.; Chao, Y.C. Duodenogastric reflux: Proposed new endoscopic classification in symptomatic patients. J. Med. Sci. 2016, 36, 1-5.

26. Taşkin, V.; Sedele, M.; Saka, O.; Kantarçeken, B. The effect of duodenogastric reflux on Helicobacter pylori presence and gastrichistopathologic changes. Turk. J. Gastroenterol. 2003, 14, 239-242. [PubMed]

27. Itoh, M.; Wada, K.; Tan, S.; Kitano, Y.; Kai, J.; Makino, I. Antibacterial action of bile acids against Helicobacter pylori and changes in its ultrastructural morphology: Effect of unconjugated dihydroxy bile acid. J. Gastroenterol. 1999, 34, 571-576. [CrossRef] [PubMed]

28. Sobala, G.M.; O'Connor, H.J.; Dewar, E.P.; King, R.F.; Axon, A.T.; Dixon, M.F. Bile reflux and intestinal metaplasia in gastric mucosa. J. Clin. Pathol. 1993, 46, 235-240. [CrossRef]

29. Szarszewski, A.; Korzon, M.; Kamiñska, B.; Lass, P. Duodenogastric reflux: Clinical and therapeutic aspects. Arch. Dis. Child. 1999, 81, 16-20. [CrossRef]

30. Tewari, S.N.; Cerezo, L.; Price, G. The prevelance of Campylobacter pylori gastritis: A study of symptomatic nonulcer dyspepsia and bile gastritis. J. Clin. Gastroenterol. 1989, 11, 271-277. [CrossRef] 
31. Matsuhisa, T.; Tsukui, T. Relation between reflux of bile acids into the stomach and gastric mucosal atrophy, intestinal metaplasia in biopsy specimens. J. Clin. Biochem. Nutr. 2012, 50, 217-221. [CrossRef]

32. Huajun, L.; Zhongyan, Z.; Yunfang, L.; Xiumei, Y.; Kaiyu, H.; Weiguo, H. Efficacy and safety of ursodeoxycholic acid in the treatment of children with bile reflux gastritis. Mod. Pract. Med. 2011, 23, 192-194.

(C) 2019 by the authors. Licensee MDPI, Basel, Switzerland. This article is an open access article distributed under the terms and conditions of the Creative Commons Attribution (CC BY) license (http://creativecommons.org/licenses/by/4.0/). 